University of Northern lowa

UNI ScholarWorks

Faculty Publications

Faculty Work

$8-2000$

\title{
Masses for Galactic Beat Cepheids
}

Noella L. D'Cruz

University of Washington

Siobahn M. Morgan

University of Northern lowa

See next page for additional authors

Let us know how access to this document benefits you

Copyright (C2000 the American Astonomical Society

Follow this and additional works at: https://scholarworks.uni.edu/ear_facpub

Part of the Earth Sciences Commons

\section{Recommended Citation}

D'Cruz, Noella L.; Morgan, Siobahn M.; and Böhm-Vitense, Erika, "Masses for Galactic Beat Cepheids" (2000). Faculty Publications. 1.

https://scholarworks.uni.edu/ear_facpub/1

This Article is brought to you for free and open access by the Faculty Work at UNI ScholarWorks. It has been accepted for inclusion in Faculty Publications by an authorized administrator of UNI ScholarWorks. For more information, please contact scholarworks@uni.edu. 
Authors

Noella L. D'Cruz, Siobahn M. Morgan, and Erika Böhm-Vitense 


\title{
MASSES FOR GALACTIC BEAT CEPHEIDS
}

\author{
Noella L. D'CruZ ${ }^{1}$ \\ Department of Astronomy, University of Washington, Box 351580, Seattle WA 98195-1580; noella@physics.usyd.edu.au \\ SIOBAHN M. MORGAN \\ Department of Earth Science, University of Northern Iowa, Cedar Falls, IA 50614-0335; morgans@ uni.edu \\ AND \\ ERIKA BÖHM-VITENSE \\ Department of Astronomy, University of Washington, Box 351580, Seattle WA 98195-1580; erica@astro.washington.edu \\ Received 1999 October 18; accepted 2000 April 24
}

\begin{abstract}
Accurate mass determinations for Cepheids may be used to determine the degree of excess mixing in the interiors of their main-sequence progenitors: the larger the excess mixing, the larger the luminosity of the Cepheid of a given mass, or the smaller the mass of a Cepheid with given luminosity. Dynamical masses determined recently for a few Cepheid binaries indicate excess mixing somewhat stronger than that corresponding to the convective overshoot models by Schaller et al. Beat Cepheids can be used similarly to test main-sequence mixing in stellar interiors. The period ratios for beat Cepheids depend on luminosity, $T_{\text {eff }}$, heavy element abundance, and mass. By comparing pulsational models and the observationally derived luminosity, $T_{\text {eff }}$, metallicities, and period ratios it is possible to obtain masses for these stars, the so-called beat masses. With the old opacities masses much smaller than the evolutionary masses were obtained. With the new OPAL opacities a beat mass close to the dynamical mass was obtained for the binary beat Cepheid Y Carinae, showing that it is now possible to obtain reliable beat masses. In this paper, we determine beat masses for seven Galactic beat Cepheids for which photometric and spectroscopic data are available. We find an average mass around $4.2 \pm 0.3 M_{\odot}$ for these stars, though the actual error limits for each star may be larger mainly because of uncertainties in $E(B-V)$ and the heavy element abundances. (As derived spectroscopically, beat Cepheids are in general metalpoor, with $-0.4 \lesssim[\mathrm{Fe} / \mathrm{H}] \lesssim 0.0$ ). The relation between the derived beat masses and the luminosities again indicates excess mixing that is somewhat larger than that corresponding to the models by Schaller et al.
\end{abstract}

Key words: Cepheids Hertzsprung-Russell (HR diagram) — stars: abundances — stars: evolution — stars: fundamental parameters - stars: interiors

\section{INTRODUCTION}

Cepheid variables have been used for many years as astronomical tools to measure distances. They form an important step in the extragalactic distance ladder (see Feast \& Walker 1987 and Feast 1999 for reviews). They also play an important role in furthering our understanding of stellar evolution and pulsations (e.g., for instance BöhmVitense 1992).

Most Cepheids pulsate in a single mode - the fundamental mode - which can yield information about their mean density via the Ritter relation, i.e., period $\propto \rho^{1 / 2}$. However, some Cepheids pulsate in more than one mode. These are called double-mode or beat Cepheids. Their period ratios can be measured very accurately and depend on their masses, luminosities, $T_{\text {eff }}$, and the abundances of the heavy elements. With the luminosities known from the periodluminosity relation, $T_{\text {eff }}$ derived from $(B-V)_{0}$ colors, and element abundances known from spectrum analysis, the socalled beat masses can be determined from the period ratios. These beat masses can be compared with the so-

\footnotetext{
${ }^{1}$ Current address: Chatterton Department of Astronomy and Department of Astrophysics, School of Physics, University of Sydney, NSW 2006 Australia.
}

called evolutionary masses, derived from the comparison of observed luminosities and those obtained from stellar evolution theory. For many years, there was a discrepancy between the beat masses (1-3 $\left.M_{\odot}\right)$ and the evolutionary masses $\left(\sim 4-7 \quad M_{\odot}\right)$. Simon (1982) suggested that this problem could be resolved if, for temperatures around $10^{5}$ $\mathrm{K}$, stellar opacities for the heavy elements were increased by a factor of 2-3 over the Cox \& Tabor (1976) opacities. Andreasen (1988) confirmed Simon's result. New opacities, the OPAL opacities derived by Iglesias \& Rogers (1996), are indeed higher by the required amount. For stellar models calculated with these new opacities, beat Cepheid masses agree approximately with evolutionary masses (Moskalik, Buchler, \& Marom 1992). It therefore appears that the new beat masses are indeed the correct masses for these Cepheids. This conclusion is supported by the study of Böhm-Vitense et al. (1997), who determined the dynamical mass for the binary beat Cepheid Y Car and found agreement with its beat mass.

The luminosity for a Cepheid with a given mass depends on the degree of mixing in the interior of its main-sequence progenitor: the larger the degree of mixing, the larger the luminosity. This means that for a given luminosity, as determined from the period-luminosity relation, a Cepheid has a smaller mass for a larger degree of mixing in its mainsequence progenitor. The empirical determination of the 
Cepheid masses therefore offers the opportunity to determine the degree of mixing in massive main-sequence stars.

For a few Cepheid binaries with blue main-sequence companions, dynamical masses of Cepheids can be determined (see, for instance, Böhm-Vitense et al. 1998 and references therein). The beat Cepheids now offer another possibility to determine additional empirical Cepheid masses and thereby get additional information about the degree of interior mixing for main-sequence stars of different masses.

In this paper, we are concerned only with beat Cepheids within our Galaxy for which heavy element abundances and $T_{\text {eff }}$ have been determined. D. Welch has compiled a list of 17 Milky Way beat Cepheids. ${ }^{2}$ Two additional stars may be beat Cepheids as well. It is likely that more of these stars exist but have not yet been discovered. These stars typically pulsate in the fundamental $(\mathrm{F})$ and first-overtone $(1 \mathrm{H})$ modes with periods $P_{0}$ and $P_{1}$, respectively. There is only one Galactic beat Cepheid, CO Aur, that pulsates in the first- and second-overtone modes (Pardo \& Poretti 1997; Poretti \& Pardo 1997). We study seven of the Galactic beat Cepheids whose photometry is available in the literature.

The Large Magellanic Cloud contains a large number of beat Cepheids. The MACHO microlensing collaboration (Alcock et al. 1995; Welch et al. 1997) reported the discovery of 76 beat Cepheids. These contain a much larger fraction of $1 \mathrm{H} / 2 \mathrm{H}$ pulsators compared with the Milky Way. The EROS microlensing survey (Beaulieu et al. 1997) has discovered 11 beat Cepheids in the Small Magellanic Cloud so far, of which seven are pulsating in the $1 \mathrm{H} / 2 \mathrm{H}$ mode, while four pulsate in the $\mathrm{F} / 1 \mathrm{H}$ mode. These stars have, however, lower than solar heavy element abundances that are not well known at present. Their $T_{\text {eff }} \mathrm{s}$ are also not yet well known, mainly because of uncertain interstellar reddening corrections. We therefore restrict our studies here to the Galactic Cepheids.

\section{MASS DETERMINATION FOR BEAT CEPHEIDS}

Period ratios of beat Cepheids can be measured to very high accuracies and depend sensitively on the masses of the Cepheids. "Beat masses" can therefore most easily be deter-

\footnotetext{
${ }^{2}$ Available at:

http://physun.physics.mcmaster.ca/Cepheid/BeatCepheid.html.
}

mined using the Petersen diagram (Petersen 1973), in which, for a given luminosity, the period ratios $P_{1} / P_{0}$ for the $1 \mathrm{H} / \mathrm{F}$ mode pulsators are plotted as a function of $\log P_{0}$ for stars with different $T_{\text {eff }}$ and different stellar masses. Theoretical periods and period ratios have been calculated, for instance, by Christensen-Dalsgaard \& Petersen (1995) and most recently by Morgan \& Welch (1997), who calculated periods and period ratios for different heavy element abundances and different stellar masses using OPAL opacities. Here we make extensive use of calculations by one of us (S. M.). Different models are well separated in the Petersen diagram. The luminosities of the beat Cepheids are known from the period-luminosity relation. With $T_{\text {eff }}$ and heavy element abundances known from photometry and spectroscopic analyses and with the observed period ratios of the beat Cepheids, we look in the Petersen diagrams for the best-fitting beat masses.

The Galactic beat Cepheids appear to have a range in metallicity that may extend from about solar to $[\mathrm{Fe} / \mathrm{H}] \sim$ -0.4 , hence we also explore the metallicity parameter space when searching for the best-fitting model. As beat Cepheids are among the lowest-period Cepheids, their masses are expected to be near the low end of the Cepheid mass range, i.e., around 4-6 $M_{\odot}$.

The Petersen diagram has been successfully used to obtain the mass of the solar metallicity binary beat Cepheid Y Car (Böhm-Vitense et al. 1997). Its dynamical mass was found to be $3.8 \pm 1 M_{\odot}$, which agrees well with its beat mass of $3.8 M_{\odot}$.

\section{EFFECTIVE TEMPERATURES, LUMINOSITIES, AND METALLICITIES}

In this paper, we have studied seven Galactic beat Cepheids for which sufficient data are available in the literature with respect to photometry, reddenings, and metallicities. Data for the beat Cepheids used here are listed in Table 1, along with the sources of the data. From left to right the columns are: star name, fundamental period, $P_{0}$, in days; first-overtone period, $P_{1}$, in days; $P_{1} / P_{0} ; \log P_{0}$; $\langle B\rangle ;\langle V\rangle$; reddening, $E(B-V)$; dereddened color, $\langle B\rangle_{0}$ $-\langle V\rangle_{0}$; effective temperature, $T_{\text {eff }}(\mathrm{K})$; spectroscopic temperature, $T_{\text {spec }}(\mathrm{K})$; the photometric temperature corresponding to the phase of spectral observations, $T_{\mathrm{ph}-\mathrm{sp}}$ and spectroscopic metallicity, $[\mathrm{Fe} / \mathrm{H}]$.

TABLE 1

Pulsational, Photometric, and Reddening Data of Beat Cepheids from the Literature

\begin{tabular}{|c|c|c|c|c|c|c|c|c|c|c|c|c|}
\hline Name & $\begin{array}{c}P_{0}^{\mathrm{a}} \\
\text { (days) }\end{array}$ & $\begin{array}{c}P_{1}^{\mathrm{a}} \\
\text { (days) }\end{array}$ & $P_{1} / P_{0}$ & $\log P_{0}$ & $\langle B\rangle$ & $\langle V\rangle$ & $E(B-V)$ & $\langle B\rangle_{0}-\langle V\rangle_{0}$ & $\begin{array}{c}T_{\text {eff }} \\
(\mathrm{K})\end{array}$ & $\begin{array}{c}T_{\text {spec }} \\
(\mathrm{K})\end{array}$ & $\begin{array}{c}T_{\mathrm{ph}-\mathrm{sp}} \\
(\mathrm{K})\end{array}$ & {$[\mathrm{Fe} / \mathrm{H}]$} \\
\hline TU Cas ...... & 2.13931 & 1.518285 & 0.709708 & 0.33027 & $8.504^{b}$ & 7.890 & $0.105^{\mathrm{c}}$ & 0.495 & 6000 & $5750^{c}$ & 5970 & $-0.43 \pm 0.31^{\mathrm{c}, \mathrm{d}}$ \\
\hline VX Pup...... & 3.0109 & 2.1390 & 0.7104 & 0.47870 & $9.250^{\mathrm{e}}$ & 8.580 & $0.165^{\mathrm{f}}$ & 0.505 & 5950 & $5900^{d}$ & 5794 & $-0.39 \pm 0.14^{\mathrm{d}}$ \\
\hline AP Vel....... & 3.12776 & 2.19984 & 0.70333 & 0.49523 & 10.983 & 10.026 & $0.560^{f}$ & 0.397 & 6450 & $6356^{\mathrm{g}}$ & 6953 & $-0.18 \pm 0.35^{\mathrm{d}}$ \\
\hline UZ Cen...... & 3.33435 & 2.35529 & 0.70637 & 0.523011 & 9.578 & 8.834 & $0.254^{\mathrm{f}}$ & 0.490 & 6050 & $6058^{\mathrm{g}}$ & 5990 & $-0.30 \pm 0.32^{\mathrm{d}}$ \\
\hline $\mathrm{AX}$ Vel ...... & 3.673170 & 2.592924 & 0.705909 & 0.56504 & 8.858 & 8.197 & $0.239^{f}$ & 0.422 & 6250 & $6348^{\mathrm{g}}$ & 6414 & $-0.43 \pm 0.29^{d}$ \\
\hline GZ Car ...... & 4.15885 & 2.93372 & 0.70542 & 0.61897 & 11.125 & 10.216 & $0.473^{\mathrm{f}}$ & 0.436 & 6250 & $6022^{\mathrm{g}}$ & 6366 & $-0.36 \pm 0.33^{\mathrm{d}}$ \\
\hline BQ Ser ...... & 4.27073 & 3.01205 & 0.7053 & 0.63050 & $11.043^{\mathrm{e}}$ & 9.619 & $0.82^{\mathrm{h}}$ & 0.604 & 5600 & $5400^{d}$ & 5893 & $-0.36 \pm 0.2^{\mathrm{d}}$ \\
\hline
\end{tabular}

a Data from Table 1, Balona (1985).

b Data for TU Cas is from Berdnikov (1992).

${ }^{c}$ Andrievsky et al. (1993).

d Andrievsky et al. (1994).

e Data for VX Pup \& BQ Ser are from Moffett \& Barnes (1984), while data for the rest stars are from Pel (1976).

${ }^{\mathrm{f}}$ Balona \& Stobie (1979).

g Barrell (1982).

${ }^{\text {h }}$ Balona \& Engelbrecht (1985). 
The pulsation period data are from Balona (1985). Pardo \& Poretti (1997) have evaluated and reanalyzed available photometric data to obtain the two periods and the harmonics that may exist. They find that periods determined earlier (e.g., Balona 1985) are in good agreement with their reanalysis.

The photometric data for TU Cas are from Berdnikov (1992). For VX Pup and BQ Ser the data are from Moffett \& Barnes (1984) and is in the Johnson system. For the rest of the stars, Walraven photometry $\left(B_{\mathrm{W}}, V_{\mathrm{W}}\right)$ of Pel (1976) was used. Standard conversions to the Johnson system were used to obtain $B, V$ magnitudes (Pel, Trefzger, \& Blaauw 1988). These are

$$
V=6.886-2.5 V_{\mathrm{w}}-0.82\left(V_{\mathrm{w}}-B_{\mathrm{w}}\right)
$$

and

$$
\begin{aligned}
(B-V)= & 2.571\left(V_{\mathrm{w}}-B_{\mathrm{w}}\right)-1.020\left(V_{\mathrm{w}}-B_{\mathrm{w}}\right)^{2} \\
& +0.500\left(V_{\mathrm{w}}-B_{\mathrm{W}}\right)^{3}-0.010 .
\end{aligned}
$$

The photometric data are used to compute average $B$ and $V$ magnitudes, $\langle B\rangle$ and $\langle V\rangle$, over the fundamental period of the Cepheid, and these are also listed in Table 1. For AX Vel, however, the Pel (1976) data correspond to that of the first overtone, because only in this star is the amplitude of the fundamental frequency smaller than that of the first overtone (Pardo \& Poretti 1997). The error in average temperature due to using this data set is about $150-200 \mathrm{~K}$, which is within our error bars as given below.

The $\langle B\rangle-\langle V\rangle$ colors were corrected for reddening by subtracting the $E(B-V)$ values from them. For TU Cas we have used the reddening value given in Andrievsky et al. (1993) and for BQ Ser the reddening given in Balona \& Engelbrecht (1985) was used. We have taken reddening values from Balona \& Stobie (1979) for the remaining five stars. The error in reddening is about 0.05 .

We used Kurucz (1979) ATLAS6 models to derive effective temperatures from the reddening corrected color, $\langle B\rangle_{0}-\langle V\rangle_{0}$. These temperatures are listed in Table 1 and have errors of about $\pm 200 \mathrm{~K}$. An error of approximately $0.05 \mathrm{mag}$ in $\langle B\rangle_{0}-\langle V\rangle_{0}$ is present because of the existence of the first overtone.

The spectroscopically determined temperatures, $T_{\text {spec }}$, are listed in Table 1 for completeness sake, as they only represent the phase at the time of observation, not an average over the period. For TU Cas, $T_{\text {spec }}$ is from Andrievsky et al. (1993), and for VX Pup and BQ Ser, $T_{\text {spec }}$ is taken from
Andrievsky et al. (1994). The rest of the spectroscopic temperatures are from Barrell (1982). Photometric temperatures, $T_{\mathrm{ph}-\mathrm{sp}}$, corresponding to the phase of observation are listed in Table 1 . They are in reasonable agreement with the spectroscopic ones for most of the stars.

The metallicity of TU Cas is obtained from the spectroscopic work of Andrievsky et al. (1993), while that of VX Pup and BQ Ser is from Andrievsky et al. (1994). For the rest of the stars, metallicities are from the spectral observations of Barrell (1982). However, the metallicities given in the table are from a reanalysis of Barrell's work by Andrievsky et al. (1993), using the WIDTH6 program. Metallicities for beat Cepheids tend to be less than solar, as shown in Table 1. (From stellar evolutionary models, it appears that mainly for lower metallicities do the blue loops extend to high enough $T_{\text {eff }}$ to reach into the domain of the beat Cepheids).

Using period-luminosity-color relations, the luminosities of beat Cepheids can be determined. We used the Schmidt (1984) $P-L-C$ relation

$$
M_{V}=-3.8 \log P+2.70\left(\langle B\rangle_{0}-\langle V\rangle_{0}\right)-2.21 .
$$

Although newer $P-L-C$ relations have been derived from Hipparcos Cepheid parallaxes (Feast \& Catchpole 1997), there is still no clear consensus as to which of the new relations is the correct one to use. The reddening data and bolometric corrections (Kurucz 1979) that we use have errors which are large enough that the errors in the derived luminosities are comparable to the differences in luminosities derived from various $P-L-C$ relations. Hence, we feel that the Schmidt relation is sufficiently accurate for our purposes. The luminosities we obtain are given in column (8) of Table 2. We estimate the errors to be about 0.1 in $\log L$

Fernie et al. (1995) maintain a database of observations for 500 Galactic Cepheids (including the beat Cepheids) at the David Dunlap Observatory (hereafter DDO database). ${ }^{3}$ We have compared the data that we used with the data in the DDO database. The comparisons for $\langle B\rangle_{0}-\langle V\rangle_{0}$; $E(B-V) ; T_{\text {eff }}(\mathrm{K}) ; \log \left(L / L_{\odot}\right)$ are listed in Table 2. Effective temperatures for AP Vel and GZ Car differ by $450 \mathrm{~K}$ or more, while that of AX Vel differs by $200 \mathrm{~K}$. This difference is largely because of the differences in the values used to

\begin{tabular}{|c|c|c|c|c|c|c|c|c|}
\hline \multirow[b]{2}{*}{$\begin{array}{c}\text { StAR NAME } \\
\text { (1) }\end{array}$} & \multicolumn{2}{|c|}{$\langle B\rangle_{0}-\langle V\rangle_{0}$} & \multicolumn{2}{|c|}{$E(B-V)$} & \multicolumn{2}{|c|}{$T_{\text {eff }}(\mathrm{K})$} & \multicolumn{2}{|c|}{$\log \left(L / L_{\odot}\right)$} \\
\hline & $\begin{array}{l}\text { LIT } \\
(2)\end{array}$ & $\begin{array}{l}\text { DDO } \\
\text { (3) }\end{array}$ & $\begin{array}{l}\text { LIT } \\
(4)\end{array}$ & $\begin{array}{l}\mathrm{DDO} \\
(5)\end{array}$ & $\begin{array}{l}\text { LIT } \\
(6)\end{array}$ & $\begin{array}{l}\text { DDO } \\
(7)\end{array}$ & $\begin{array}{l}\text { LIT } \\
(8)\end{array}$ & $\begin{array}{l}\text { DDO } \\
(9)\end{array}$ \\
\hline TU Cas & 0.495 & 0.490 & 0.105 & 0.115 & 6000 & 6000 & 2. 74 & 2.74 \\
\hline VX Pup...... & 0.505 & 0.488 & 0.165 & 0.136 & 5950 & 6000 & 2. 95 & 3.00 \\
\hline AP Vel....... & 0.397 & 0.504 & 0.560 & 0.515 & 6450 & 6000 & 3. 07 & 2.97 \\
\hline UZ Cen................ & 0.490 & 0.498 & 0.254 & 0.275 & 6050 & 6000 & 3. 03 & 3.11 \\
\hline AX Vel & 0.422 & 0.478 & 0.239 & 0.224 & 6250 & 6050 & 3. 16 & 3.11 \\
\hline GZ Car ...... & 0.436 & 0.571 & 0.473 & 0.419 & 6250 & 5750 & 3. 23 & 3.09 \\
\hline BQ Ser ....... & 0.604 & 0.567 & 0.820 & 0.841 & 5600 & 5750 & 3. 11 & 3.14 \\
\hline
\end{tabular}

${ }^{3}$ Available at: http://ddo.astro.utoronto.ca/cepheids.html.

TABLE 2

COMPARISON BETWEen LITERATURE USED (LIT) AND DDO CePheID DATABase 
derive the $\langle B\rangle_{0}-\langle V\rangle_{0}$ color from the literature and the DDO database. Since it is not clear which temperatures are the correct ones to use, we have used both to derive stellar parameters for these three stars.

\section{DERIVING STELLAR PARAMETERS FROM THE PETERSEN DIAGRAM}

The Cepheid models used in this paper are computed with the code described in Morgan \& Welch (1997). OPAL opacities are used in the model calculations. Models were initially computed for five different metallicities, namely, for $Z=0.01,0.0125,0.015,0.0175$, and 0.02 , where $Z$ is the mass fraction of heavy elements. Effective temperatures for the models range from 5600 to $6600 \mathrm{~K}$. Luminosities are in the range $2.6 \leq \log \left(L / L_{\odot}\right) \leq 3.3$. A representative set of models are shown in the Petersen diagram in Figure 1 for a metallicity of $Z=0.015$. Lines join models with the same luminosity and mass, both values of which are given next to the curves. Open symbols are for a mass of $3.5 M_{\odot}$, while filled symbols are for $4.5 M_{\odot}$ models. Curves for luminosities of $\log L / L_{\odot}=2.8,3.0$, and 3.2 are shown. Effective temperature for the models increases from 5600 to $6600 \mathrm{~K}$ in steps of $100 \mathrm{~K}$ from right to left, with some values marked on the $\log L / L_{\odot}=3.2,3.5 M_{\odot}$ curve. It can be seen that luminosity increases diagonally toward the bottom right. Models of lower mass have smaller period ratios and larger fundamental periods for $\log P_{0} \lesssim 0.4$ at constant luminosity. For $\log P_{0}>0.4$, period ratios are similar, while for a given luminosity periods are larger, as mass decreases.

In Figure 2 we show where models for $3.5 M_{\odot}$ stars with different metallicities $(Z=0.015,0.0125)$ and luminosity lie in the Petersen diagram. Models of the same effective temperature and luminosity have lower period ratios as metallicity increases, while the fundamental period remains approximately the same.

The beat Cepheids are compared with models in the Petersen diagram in Figure 3. Since it is possible for different combinations of the stellar parameters (mass, lumi-

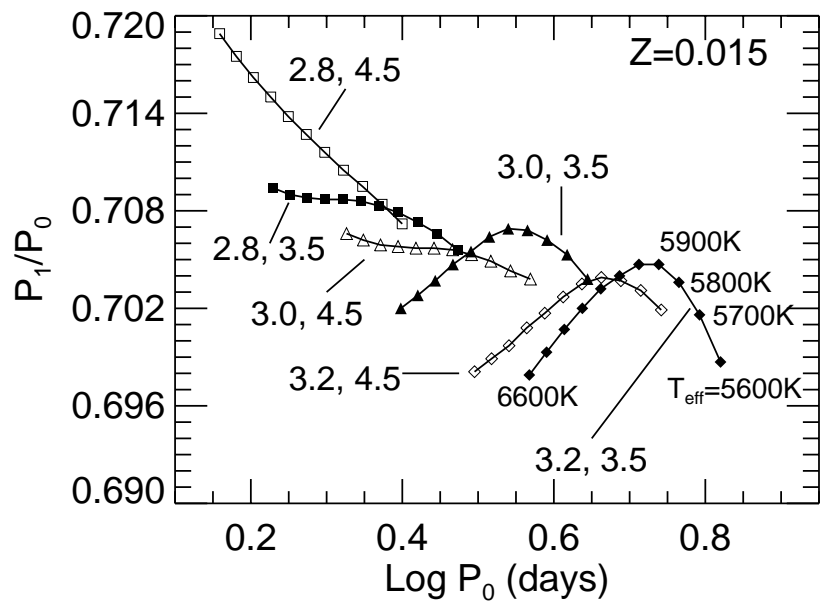

FIG. 1.-Petersen Diagram for Cepheid models with $Z=0.015$. The lines join models with the same luminosity and mass, both of whose values are given next to each curve. Solid symbols are for $3.5 M_{\odot}$, and open symbols correspond to $4.5 M_{\odot}$. Squares, triangles, and diamonds correspond to $\log \left(L / L_{\odot}\right)=2.8,3.0$, and 3.2, respectively. Effective temperatures run from $5600-6600 \mathrm{~K}$ in steps of $100 \mathrm{~K}$ from right to left, and a few of the temperatures are marked on the $\log L=3.2, M=3.5$ curve.

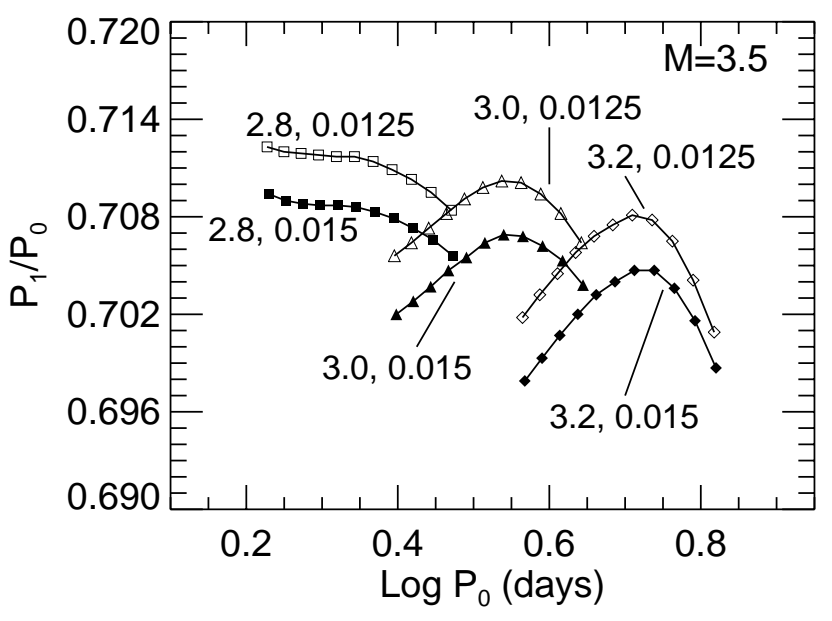

FIG. 2.-Petersen Diagram for Cepheid models with $M=3.5 M_{\odot}$. The lines join models with the same luminosity and metallicity, both of whose values are given next to each curve. Open symbols are for $Z=0.0125$, and solid symbols correspond to $Z=0.015$. Squares, triangles, and diamonds correspond to $\log \left(L / L_{\odot}\right)=2.8,3.0$, and 3.2. Effective temperatures run from $5600-6600 \mathrm{~K}$ in steps of $100 \mathrm{~K}$ from right to left for each curve. One can note that at a given effective temperature, mass, and luminosity, a decrease in metallicity corresponds to a increase in the period ratio, $P_{1} / P_{0}$.

nosity, temperature, and metallicity) to produce models with the observed period and period ratios of a given beat Cepheid, certain of these parameters must be initially set to either well-observed values or constrained to a limited range of values. The effective temperature was constrained to within $\pm 200 \mathrm{~K}$ of the literature based values given in Table 2, though for some stars, the DDO values were also used. The luminosities were initially set to the Schmidt values given in Table 2, but were later allowed to vary slightly, generally less than 0.05 . Initial values for mass and metallicity were obtained from the series of models described above. These values were then adjusted gradually to produce models with the correct period and period ratios. In general, there is not a great deal of flexibility in the metallicity values, typically only variations of 0.0025 or less produce models with the appropriate period and period ratios, regardless of the other parameters within their uncertainty range. This strong dependence on the metallicity value tends to constrain it as well, leaving the mass as the only unknown. If for given values of $\log L / L_{\odot}$ and $T_{\text {eff }}$ there are no models that produce the correct period and period ratio, regardless of the values of mass and metallicity, we search for values of $T_{\text {eff }}$ and $\log L / L_{\odot}$ within the given error limits. Those with the smallest differences from the predetermined values are taken as the best-fit values for the period and period ratio. If there is a range of values for the $T_{\text {eff }}$ and $\log L / L_{\odot}$ for which the observed period and period ratio can be obtained, then this can produce some uncertainty in the value for the mass. In the case of a typical beat Cepheid whose $\log \left(L / L_{\odot}\right)=3.0$ and $T_{\text {eff }} \sim 6200 \mathrm{~K}$, and period and metallicity are fixed, a change of $\Delta T_{\text {eff }} \sim 100 \mathrm{~K}$ corresponds to about $\Delta M \sim 0.3 M_{\odot}$. If the period, temperature, and metallicity values are kept fixed, then a change of $\Delta \log \left(L / L_{\odot}\right) \sim 0.01$ results in $\Delta M \sim 0.1 M_{\odot}$.

Both Figure 3 and Table 3 list the best-fit stellar parameters. Table 3 includes the corresponding $[\mathrm{Fe} / \mathrm{H}]$ values as well. For the three stars whose colors from the DDO database differed from the literature-based values, we list best- 


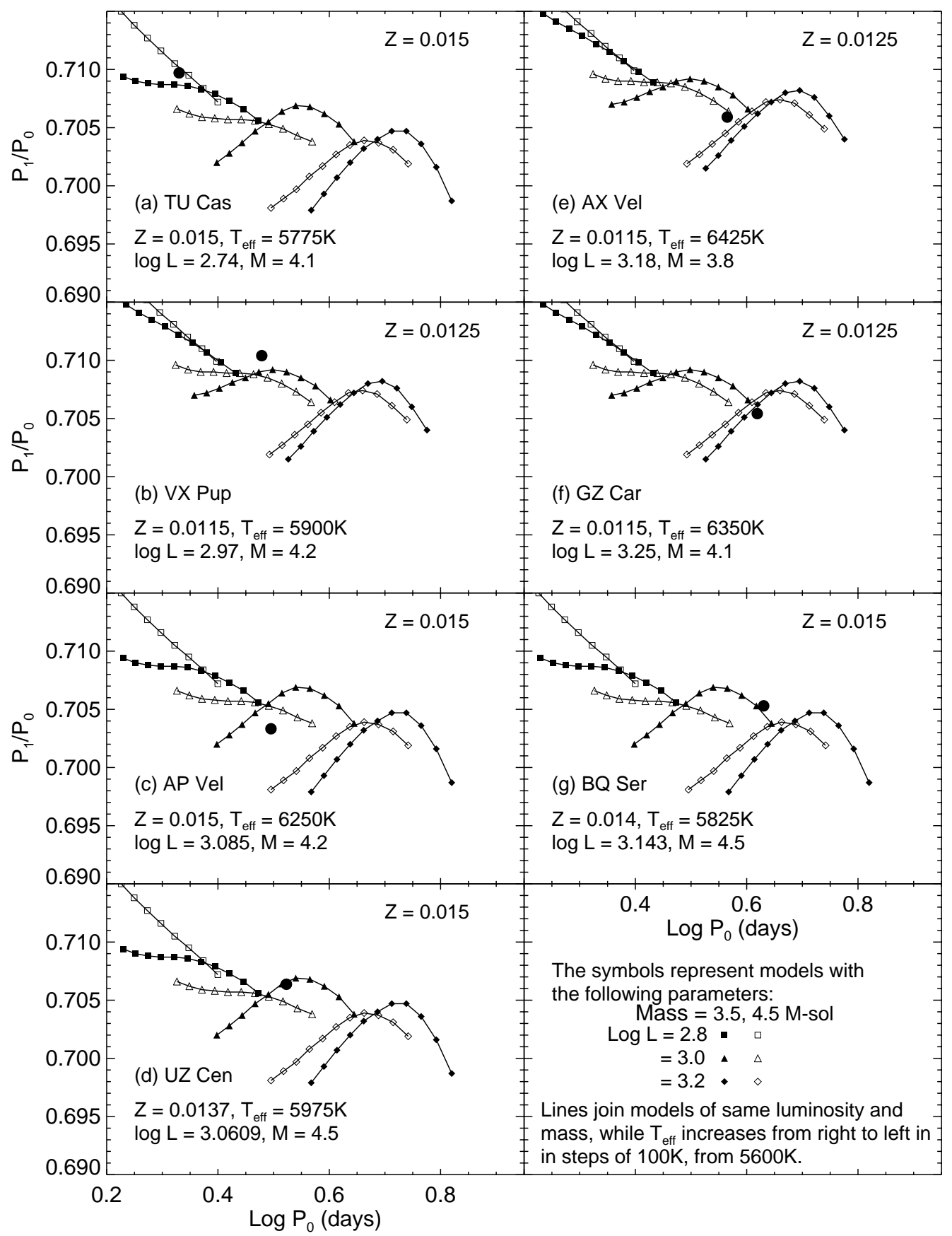

FIG. 3. - Petersen diagram for the seven beat Cepheids considered in the paper in relation to the Cepheid models. Each panel shows models for masses of 3.5 and $4.5 M_{\odot}$ and for the metallicity closest to the best-fitting one, which is either $Z=0.015$ or 0.0125 . The symbols used in the plots are the same as that of Fig. 1; the bottom right corner has a short explanation of the symbols. Each panel has the beat Cepheid shown by a solid dot and the best-fitting parameters are given in the bottom left corner of the panel.

fitting values as well. The masses of the best-fit models seem to be confined to a narrow range of $4.2 \pm 0.35 M_{\odot}$ and are at the lower end of the Cepheid mass range, as expected.

It can be seen that AP Vel's best-fit effective temperatures and metallicities are almost identical, but since luminosity differs by 0.035 dex, the resulting mass changes by about $10 \%$. In the case of AX Vel and GZ Car, there is also a difference of about $10 \%$ in the derived masses, although effective temperatures differ by about $500 \mathrm{~K}$, and luminosities and metallicities vary by $25 \%-40 \%$. It appears that the final masses are uncertain by about $10 \%$.
The period ratios seem to require metallicities around $[\mathrm{Fe} / \mathrm{H}] \sim-0.11$ to achieve the best fit. These abundances are systematically higher than those determined spectroscopically but are within the error bars. Spectroscopic abundances would yield not as good a fit for the same effective temperature and luminosity. As the resolution of models in the Petersen diagram is large, metallicity is constrained to a much smaller range than is possible to measure from spectra. Hence, the metallicities determined here should be quite accurate, provided the theoretical opacities and models are sufficiently accurate. If so, the Peter- 
TABLE 3

Best-Fitting Model Parameters

\begin{tabular}{|c|c|c|c|c|c|}
\hline Name & $\begin{array}{l}T_{\text {eff }} \\
(\mathrm{K})\end{array}$ & $\log \left(L / L_{\odot}\right)$ & $Z$ & {$[\mathrm{Fe} / \mathrm{H}]$} & $\begin{array}{l}\text { Mass } \\
\left(M_{\odot}\right)\end{array}$ \\
\hline TU Cas........... & 5775 & 2.74 & 0.015 & -0.05 & 4.1 \\
\hline VX Pup ........... & 5900 & 2.97 & 0.0115 & -0.17 & 4.2 \\
\hline AP Vel $\ldots . \ldots \ldots \ldots$ & 6250 & 3.085 & 0.015 & -0.05 & 4.2 \\
\hline AP Vel (DDO) ... & 6250 & 3.05 & 0.0155 & -0.04 & 3.8 \\
\hline UZ Cen ........... & 5975 & 3.061 & 0.0137 & -0.09 & 4.5 \\
\hline AX Vel ............ & 6425 & 3.18 & 0.0115 & -0.17 & 3.8 \\
\hline AX Vel (DDO) ... & 5900 & 3.08 & 0.014 & -0.08 & 4.4 \\
\hline GZ Car........... & 6350 & 3.25 & 0.0115 & -0.17 & 4.1 \\
\hline GZ Car (DDO)... & 5825 & 3.12 & 0.015 & -0.05 & 3.9 \\
\hline BQ Ser...$\ldots \ldots \ldots$ & 5825 & 3.143 & 0.014 & -0.08 & 4.5 \\
\hline
\end{tabular}

NoTE.-Since effective temperatures for AP Vel, AX Vel, and GZ Car from the literature and the DDO database differed, the final set of parameters for both temperatures are listed here.

sen diagram will be an extremely useful tool for determining metallicities, for instance, of the Magellanic Cloud beat Cepheids.

\section{BEAT CEPHEIDS IN THE H-R DIAGRAM}

In the H-R diagram, Cepheids are expected to be found mostly at the hot end of the blue loops where evolution proceeds more slowly than during the rest of the loop (see, for instance, Böhm-Vitense et al. 1997). The extent of blue loops increases for lower metallicities and decreases for increasing convective overshoot. As mentioned in the introduction, convective overshoot affects the luminosity of the blue loops for a given mass: the greater the extent of mixing in the interiors of the main-sequence progenitors the larger the increase in luminosity of the Cepheids. Hence, a good mass determination for the Cepheids determines the degree of mixing in the interiors of massive main-sequence stars.

Figure 4 shows the Hertzsprung-Russell diagram for the double-mode Cepheids, along with stellar evolutionary tracks for different masses and different assumptions about convective overshoot mixing, as well as different metallicities. best-fitting effective temperatures and luminosities for the beat Cepheids are plotted, with the stars shown as solid dots in all panels. The stars are labelled in the middle panel. For AP Vel, AX Vel, and GZ Car we have plotted the lower DDO luminosities. In the top panel, solar metallicity tracks for 4 and $5 M_{\odot}$ stars from Bressan et al. (1993) for classical mixing (dotted lines) are shown and also for models with different degrees of convective overshoot. For the Schaller et al. (1992) models (dashed lines) convective core overshoot distance was derived assuming a ratio of mixing length to pressure scale height of 0.4 for the convective core. For the Bressan et al. (1993) overshoot models (solid lines) a ratio of mixing length to pressure scale height of 0.5 was assumed to derive the overshoot distance. It can be seen that Galactic beat Cepheids are hotter and brighter than the solar metallicity tracks due to their lower metallicities.

The middle panel of Figure 4 contains 4 and $5 M_{\odot}$ tracks with convective overshoot for solar and LMC metallicities (dotted and solid lines, respectively, from Bressan et al. 1993). This panel shows that for the $4 M_{\odot}$ stars, the blue loops for metal-poor stars span a luminosity range of $\Delta \log L \sim 0.2$. We may expect a similar spread in the observed luminosities for Cepheids with a given mass, as may be obvious from Table 3. However, the masses that we determine have

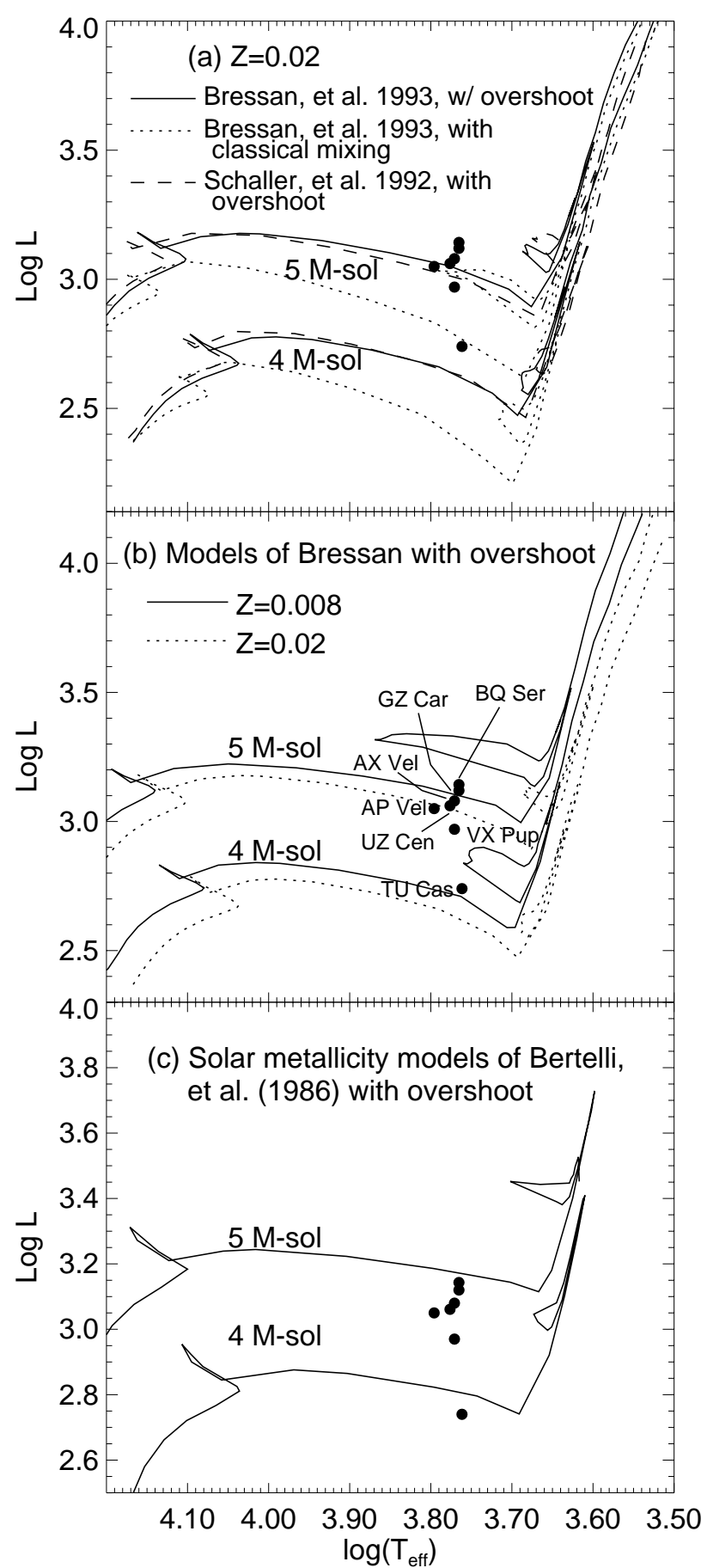

FIG. 4.-H-R Diagram showing evolutionary tracks of 4 and $5 M_{\odot}$ stars for different models, along with the best-fit values for the beat Cepheids. In all panels, beat Cepheids are shown by the solid dots. For AP Vel, AX Vel, and GZ Car we plot the lower DDO luminosities. Solar metallicity tracks are shown in panel $(a)$ : solid lines correspond to the Bressan et al. (1993) models with overshoot; dotted lines are for classical mixing models by the same authors; dashed lines are from Schaller et al. (1992) with overshoot. Panel (b) contains tracks for Bressan et al. (1993) overshoot models for metallicities of $Z=0.008,0.02$, in solid and dotted lines, respectively. Convective overshoot in the top two panels is about 0.25 pressure scale height. It seems that the beat Cepheids tend to lie near the blue end of the blue loops. If convective overshoot is increased for lower metallicity models, better agreement will result between pulsational and evolutionary masses. This is shown in panel $(c)$, where the solar metallicity evolutionary tracks of Bertelli et al. (1986) for an convective overshoot distance of half a pressure scale height are plotted. For these models, the positions of the beat Cepheids with an average mass of $4.2 M_{\odot}$ fit on the extended loop for the $4 M_{\odot}$ stars. Please refer to the text for more details. 
estimated uncertainties of about $0.4 M_{\odot}$ (due to the uncertainties in the heavy element abundances and the $T_{\text {eff }}$ ), so the spread in luminosities would be slightly larger. From the figure, it can be seen that TU Cas is clearly separated from the remaining six stars by about $\Delta \log L \sim 0.25$. If our data are correct, then TU Cas may possibly be a first crossing star. For the remaining stars the average mass is about $4.22 M_{\odot}$, and the average luminosity about $\log \left(L / L_{\odot}\right)=3.1$. This is larger than the $\log \left(L / L_{\odot}\right)$ expected for a $4.22 M_{\odot}$ star according to the Bressan et al. evolutionary tracks. It therefore appears that somewhat more excess mixing than the one assumed for the Bressan or Schaller models is necessary to find agreement for the beat Cepheids, unless the OPAL opacities still need an upward revision (which would lead to higher beat masses).

For the star Y Carinae, Böhm-Vitense et al. (1997) also found that more excess mixing was required than assumed by the Schaller models. For this beat Cepheid they found best agreement with the larger convective overshoot models of Bertelli et al. (1986), who assumed a mixing length of one pressure scale height for the convective core to calculate the overshoot distance for the upper main-sequence stars in their models.

Böhm-Vitense et al. (1998 and references given there) determined dynamical masses of classical Cepheids, which indicate excess mixing corresponding to convective overshoot that probably lies between that assumed in the Schaller et al. (1992) or Bressan et al. (1993) models and the Bertelli et al. (1986) models. Those classical Cepheids have masses between 5 and $6 M_{\odot}$. Studies of bump Cepheids in the LMC also indicate that similar large convective overshoot is needed to reconcile pulsation and evolutionary masses around 4.5-5 $M_{\odot}$ (Wood, Arnold, \& Sebo 1997; Wood 1998). Here we reach the same conclusion for the beat Cepheids with masses around $4 M_{\odot}$.

In order to better quantify the excess mixing in the beat Cepheids, we have compared the Bertelli et al. (1986) tracks for a heavy element abundance of $Z=0.02$ with the beat Cepheids in the bottom panel of Figure 4. From Figure $4 b$ we can see that the lower metal abundance of the beat Cepheids would lead to somewhat higher luminosities for the models. The Bertelli et al. calculations use the Cox Tabor opacities. The higher OPAL opacities, on the other hand, lead to somewhat lower luminosities for stellar models (see Bressan et al. 1993). The two "effects" therefore nearly cancel. The tracks shown in Figure $4 c$ are therefore expected to be fairly accurate except that the blue loops would extend to higher temperatures for the lower metallicity of the beat Cepheids. Figure $4 c$ shows that for these models the position of the beat Cepheids in the H-R diagram would fit on the extended loops for $4 M_{\odot}$ stars and would be an indication of rather large excess mixing for the $4 M_{\odot}$ beat Cepheid progenitors. Again the uncertainties are too large to reach a firm conclusion. Our data are also not accurate enough to determine a possible mass dependence of the excess mixing.

\section{SUMMARY}

We have determined "beat" masses from the fundamental period and ratios of the periods of seven Galactic beat Cepheids for which photometric and spectroscopic data are available. To determine the masses of the stars we compare them with beat Cepheid models in the Petersen diagram where models of different effective temperature, luminosity, mass and metallicity are well separated. Böhm-Vitense et al. (1997) had successfully used this method to obtain the beat mass of the binary beat Cepheid Y Car. Its beat mass of 3.8 $M_{\odot}$ compares well with their determination of its dynamical mass of $3.8 \pm 1 M_{\odot}$.

We find beat Cepheids masses to range between 3.8-4.5 $M_{\odot}$ and metallicities of $[\mathrm{Fe} / \mathrm{H}] \sim-0.11$ for the best-fitting models. These masses are at the lower end of the Cepheid mass range, as would be expected from the stars' shorter pulsation periods. The metallicities we determine from the fitting procedure are higher than the ones obtained from earlier spectra, but are within the spectroscopic error bars. Since the metallicity of the best-fitting models is tightly constrained, we assume that our values are sufficiently accurate for the data we use.

We compared the beat Cepheids with stellar evolutionary tracks of Bressan et al. (1993) and Schaller et al. (1992) to assess the degree of interior mixing that existed on the main sequence. For the current data set, we find that for $4 M_{\odot}$ stars, the excess mixing must be larger than that assumed for the Bressan or Schaller models. The agreement of the dynamical and beat mass for Y Carinae (Böhm-Vitense et al. 1997) supports this assertion. Excess mixing larger than the one assumed by Bressan and Schaller was also inferred by Böhm-Vitense et al. (1998), who determined dynamical masses of classical Cepheids, as well as by Wood (1998) for bump Cepheids in the Large Magellanic Cloud. We compare the $4 M_{\odot}$ beat Cepheids with the evolutionary tracks of Bertelli et al. (1986) which have larger excess mixing and find reasonable agreement with the $4 M_{\odot}$ tracks.

The location of TU Cas relative to the other six beat Cepheids seems to indicate that it is a first crossing star.

Improved data for the beat Cepheids will provide better constraints on the masses, the extent of convective overshoot, and its dependence on mass. Our understanding of the evolutionary and pulsational properties of these stars will be bettered by studies of the abundant Magellanic Clouds beat Cepheid population.

This research was supported by NASA grant GO4541.01-92A to E. B.-V., which is gratefully acknowledged. This paper made use of stellar models from the CDS Catalogue database. Some models were kindly sent to us in electronic form by G. Bertelli. We thank the anonymous referee for comments that helped to significantly improve the paper.

\section{REFERENCES}

Alcock, C. et al. 1995, AJ, 109, 1653

Andreasen, G. K. 1988, A\&A, 201, 72

Andrievsky, S. M., Kovtyukh, V. V., Makarenko, E. N., \& Usenko, I. A. 1993, MNRAS, 265, 257

Andrievsky, S. M., Kovtyukh, V. V., Usenko, I. A., Klochkova, V. G., \& Galazutdinov, G. A. 1994, A\&AS, 108, 433

Beaulieu, J. P., et al. 1997, A\&A, 321, L5
Balona, L. A. 1985, in Cepheids: Theory and Observations, ed. B. Madore (Cambridge: Cambridge Univ. Press), 17

Balona, L. A., \& Engelbrecht, C. A. 1985, Inf. Bull. Variable Stars, No. 2758

Balona, L. A., \& Stobie, R. S. 1979, MNRAS, 189, 659

Barrell, S. 1982, MNRÄS, 200, 127

Berdnikov, L. N. 1992, Soviet Astron. Lett., 18, 130

Bertelli, G., Bressan, A., Chiosi, C., \& Angerer, K. 1986, A\&AS, 66, 191 
Böhm-Vitense, E. 1992, Introduction to Stellar Astrophysics, Volume 3 (Cambridge: Cambridge Univ. Press)

Böhm-Vitense, E., Evans, N. R., Carpenter, K., Albrow, M. D., Cottrell, P.L. Robinson, R., \& Beck-Winchatz, B. 1998, ApJ, 505, 903

Böhm-Vitense, E., Evans, N. R., Carpenter, K., Morgan, S., BeckWinchatz, B., \& Robinson, R. 1997, AJ, 114, 1176

Bressan, A., Fagotto, F., Bertelli, G., \& Chiosi, C. 1993, A\&AS, 100, 647

Christensen-Dalsgaard, J., \& Petersen, J. O. 1995, A\&A, 299, L17

Cox, A. N., \& Tabor J. E. 1976, ApJS, 31, 271

Feast, M. 1999, PASP, 111, 775

Feast, M. W., \& Catchpole, R. M. 1997, MNRAS, 286, L1

Feast, M. W., \& Walker, A. R. 1987, ARA\&A, 25, 345

Fernie, J.D., Beattie, B., Evans, N.R., \& Seager, S. 1995, Inf. Bull. Variable Stars, No. 4148

Iglesias, C. R., \& Rogers, F. J. 1996, ApJ, 464, 943

Kurucz, R. L. 1979, ApJS, 40, 1
Moffett, T. J., \& Barnes, T. G., III. 1984, ApJS, 55, 389

Morgan, S. M., \& Welch, D. L. 1997, AJ, 114, 1183

Moskalik, P., Buchler, J. R., \& Marom, A. 1992, ApJ, 385, 685

Pardo, I., \& Poretti, E. 1997, A\&A, 324, 121

Pel, J. W. 1976, A\&AS, 24, 413

Pel, J. W., Trefzger, Ch. F., \& Blaauw, A. 1988, A\&AS, 75, 29

Petersen, J. O. 1973, A\&A, 27, 89

Poretti, E., \& Pardo, I. 1997, A\&A, 324, 133

Schaller, G., Schaerer, D., Meynet, G., \& Maeder, A. 1992, A\&AS, 96, 269

Schmidt, E. G. 1984, ApJ, 285, 501

Simon, N. R. 1982, ApJ, 260, L87

Welch, D., et al. 1997, in IAP Colloq., Astrophysical Returns of Microlensing Surveys, ed. R. Ferlet, J. P. Maillard, \& B. Raban, 205

Wood, P. R. 1998, in Views on Distance Indicators, ed. P. Caputo, Mem Soc. Astron. Italiana, 69, 99

Wood, P. R., Arnold, A. S., \& Sebo, K. M. 1997, ApJ, 485, L25 